To appear in December 1997 A.J.

\title{
Low-mass Binaries in the Hyades - A scarcity of brown dwarfs
}

\author{
I. Neill Reid and John E. Gizis \\ Palomar Observatory, 105-24, California Institute of Technology, Pasadena, CA 91125, \\ e-mail: inr@astro.caltech.edu,jeg@astro.caltech.edu
}

\begin{abstract}
We have obtained HST Planetary Camera observations of a total of fifty-three low-mass $\left(M<0.3 M_{\odot}\right)$ members of the Hyades cluster. Nine of these stars are resolved as binaries, with separations between 0.1 and 3.1 arcseconds, while a further three are probably equal-mass systems at smaller separations. Allowing for observational selection effects, this corresponds to an observed binary fraction of $11.3 \pm 4.6 \%$ for systems with separations in the range 14 to 825 a.u., consistent with observations of solar neighbourhood M-dwarfs. The mass-ratio distribution is only consistent marginally with the secondary stars being drawn from a $\Psi(M) \propto M^{-1}$ mass function, with three equal-mass systems amongst the six binaries with observed separations in the régime where our observations extend below the hydrogen-burning limit. Considering the entire sample, the absence of any brown dwarf companions amongst our sample makes it unlikely that the mass function of stellar/brown dwarf companions rises as steeply as $\mathrm{M}^{-1}$. If the Hyades has an age of $\sim 600$ Myrs, our results are consistent only at the $2 \sigma$ level with a flat $\left(\mathrm{M}^{0}\right)$ mass function.
\end{abstract}

Subject headings: binaries: general; stars: low mass; stars: luminosity function

\section{Introduction}

It is by now well established that approximately half of the stellar systems in the Galactic disk consist of at least two stars. This high frequency of multiple stars suggests

\footnotetext{
${ }^{1}$ Based on observations with NASA/ESA Hubble Space Telescope, obtained at the Space Telescope Science Institute, which is operated by AURA, Inc., under NASA contract NAS5-26555
} 
that binary formation is an integral part of the overall star-formation process, rather than separate, post-formation event. Thus, an empirical determination of parameters such as the overall binary frequency, the mass-ratio distribution and the variation in those quantities as a function of the mass of the primary star, can constrain formation mechanisms. Moreover, binary star surveys represent one of most effective methods of searching for objects of substellar mass, and hence setting limits on the likely numbers of brown dwarfs.

Most statistical studies of binaries have concentrated on field stars in the immediate solar vicinity. In particular, Duquennoy \& Mayor (1991) conducted a detailed spectroscopic survey of 164 late-F and G-dwarfs drawn from the Nearby Star Catalogue (Gliese, 1969; Gliese \& Jahreiss, 1979). Combining the spectroscopic binaries detected from those observations with data from the literature on binaries at wider separations, they estimate a binary frequency of at least $60 \%$ amongst solar-type stars. In contrast, surveys of lower-mass stars (Marcy \& Benitz, 1989, Henry \& McCarthy, 1990, Simons et al., 1996) indicate a lower overall binary frequency of $\sim 35 \%$. Moreover, Reid \& Gizis (1997) have argued that the mass ratio distribution amongst M-dwarfs differs significantly from that amongst the higher-mass G-dwarfs, with a clear bias towards equal-mass systems amongst the low-mass stars. This conclusion rests upon observations of a total of barely $75 \mathrm{M}$-dwarfs, however.

Open clusters provide an alternative avenue for studying binary statistics. One has the advantage of a sample of comparable size to that provided by the nearest stars, but comprising stars of known age and abundance, formed in the same environment. One is, however, faced with the potential drawback that dynamical evolution may have influenced either (or both) the relative binary fraction and the distribution of orbital parameters. The latter issue can be addressed by combining observations of stars in clusters spanning a range of ages.

Amongst the nearer open clusters, the Hyades, at an average distance of 48 parsecs, is clearly a prime target. The proximity of the cluster and the significant space motion relative to the Sun lead to substantial proper motions, and surveys by van Buren (1952), Pels et al. (1975), Lutyen et al. (1981), Schwann (1991), Reid (1992) and Bryja et al. (1994) have provided a well-defined list of cluster members to $\mathrm{V}=19.5$ or $\mathrm{M}_{V} \sim 16$. Radial velocity surveys for spectroscopic binaries have been undertaken, primarily by Griffin et al. (1988), while high-resolution imaging surveys for companions at wider separations have been carried out at optical (Mason et al., 1993) and infrared (Patience et al., 1997) wavelengths. These surveys, however, are restricted to the brighter stars in the cluster, scarcely extending fainter than K-type dwarfs. Thus, while the results provide a valuable dataset for comparison with the Duquennoy \& Mayor analysis, there is little overlap with the local surveys of low-mass stars. 
Over the last three years, we have been carrying out a survey aimed specifically at identifying binary companions at moderate to wide separations amongst the lowest-mass members of the Hyades cluster. We have been using the Planetary Camera on the Hubble Space Telescope to obtain high spatial-resolution images which are capable of detecting secondary companions at separations of 15 a.u. or more, and with masses at or below the hydrogen-burning limit. Preliminary results from this survey were reported in Gizis \& Reid (1995) (hereinafter paper I). Observations have since been made of a further 39 candidate Hyads, more than doubling the sample size. Section 2 describes those observations; in section 3, we calculate the binary frequency and compare our result against observations of higher-mass Hyades stars, and data for stars in the Solar Neighbourhood; section 4 discusses the implications of our lack of detection of any candidate brown dwarf companions; section 5 presents our conclusions.

\section{Observations}

\subsection{HST Planetary Camera data}

All of the stars in the present sample are selected from the region of the Hyades covered by the Reid (1992) survey. All have VRI photometry (Reid, 1993) and absolute magnitudes of $\mathrm{M}_{V} \geq+11.9$. Table 1 lists photometry and (for Hyades stars) distance estimates for the thirty-nine stars added to the sample since the observations reported in paper I. All save one of these stars are drawn from the Reid (1992) proper-motion survey: the exception is B 804, from Bryja et al. (1994), which is the lowest-luminosity Hyades member identified to date. The distances are derived from the observed proper motion, assuming that the stars are Hyades members. The Reid (1992) motions are absolute, calibrated using a galaxian reference frame, and there is good agreement between the mean cluster motion derived from that study and Schwann's (1991) meridian circle results. On that basis, we have adopted the Schwann determination of the convergent point and the Detweiler et al. (1984) cluster radial velocity measurement for these calculations; our previous analysis was based on the Gunn et al. (1988) convergent point. Schwann's analysis leads to a distance modulus of 3.40 magnitudes (47.9 parsecs) to the cluster centre, and provides a distance-scale zeropoint accurate to $\sim 3 \%$. Distances derived for individual stars using this method are independent of the photometry, and the $\sim 0^{\prime \prime} .005$ uncertainties in the proper motions of these faint stars correspond to $\mathrm{a} \sim 5 \%$ uncertainty in the distance.

Each star was centred in the Planetary Camera (PC) on HST, and observed using the F850LP filter with exposure times of from 3 to 70 seconds. The latter were specified to leave the target star unsaturated, allowing a direct measurement of the relative brightness 
of any potential companions detected. Two images were taken of most stars from our Cycle 4 allocation and of all stars observed during Cycle 6, allowing unambiguous elimination of cosmic-ray events. The PC has a plate-scale of $0.046 \operatorname{arcsec}_{\text {pixel }}{ }^{-1}$, while the diffraction limit of HST at 8500 Ais 0.073 arcseconds. As described in Reid \& Gizis (1997), we have used both the DAOFIND routines and Lucy-Richardson deconvolution techniques to search for binary companions. These methods are capable of detecting an equal-luminosity companion at a separation of 0.09 arcseconds, and companions fainter by $\Delta I$ of 1,3 and 5 magnitudes at separations of $0.14,0.23$ and 0.31 arcseconds, respectively. Three stars in the current sample (RHy 126, 309 and 391) appear to have significant elongation in our PC observations, suggesting that they are probably equal-mass binaries with angular separations of $\sim 0.1$ arcseconds. Table 1 lists our estimates of $\rho$ and $\Delta I$ for those three stars, where we have used DAOPHOT point-spread function fitting to deconvolve the HST images. The dates of each binary-star observation are listed in Table 1, and the images are available in the HST archive.

Four of the stars in the present sample are identified as background field stars, rather than Hyades cluster members. As Table 1 show, all four stars have formal membership probabilities of less than $20 \%$ and, as Hyades members, lie towards the lower edge of the colour-magnitude relation (Reid, 1993). (As emphasised above, the distances, and hence absolute magnitudes, are derived from the observed proper motion under the assumption that the transverse velocity matches that of the cluster.) Two stars, RHy 80 and 271, lack respectively the chromospheric and coronal activity which characterises cluster members?. The other two stars, RHy 77 and 110, are multiple systems, and are rejected as cluster members based on their location in the H-R diagram after allowing for the contribution to the ground-based photometry made by the secondary star (Figure 1a). RHy 77 is a near equal-mass binary, separation 0.46 arcseconds, while RHy 110 is a triple system, with components of $\mathrm{I}=15.32,16.21$ and $17.00 \mathrm{mag}\left(\rho(\mathrm{AB})=0^{\prime \prime} 69, \Delta(\mathrm{BC})=0^{\prime \prime} 17\right)$.

The widest binary listed in Table 1 has a separation of 1.12 arcseconds. None of these binaries were resolved in ground-based observations, and with HST observations at only one epoch, we cannot identify these stars conclusively as bound companions. However,

\footnotetext{
${ }^{2}$ We note that RHy 225 , with a membership probability of $89 \%$, and included as a cluster member in paper I, also lacks $\mathrm{H} \alpha$ emission, and is likely to be a non-member. Cluster membership probability is based on comparing the stellar proper motion against a model for the expected cluster distribution and the proper motions of the underlying field star. Observationally, we deal with individual stars, rather than distributions, and there is nothing to prevent a field star having exactly the motion expected for a cluster member. Thus it is essential that proper motion-selected stars be regarded as only candidate cluster members, regardless of the formal membership probability, until confirmed by independent photometric, chromospheric and radial velocity criteria.
} 
the observed surface density of stars with $14<\mathrm{I}<17$ magnitude is only $\sim 1600 \mathrm{deg}^{-2}$ in the Hyades fields surveyed by Reid (1992), implying an average separation of 3 arcminutes between stars, and a probability of $\sim 6 \times 10^{-4}$ of finding two stars at 1.2 arcseconds separation. All of the resolved binaries listed in Table 1 are therefore very likely to be bound systems.

Possible companions at wider separations are accessible to ground-based CCD and photographic observations, notably POSS I and POSS II plate material. All of the potential wide binary companions detected in the PC observations are visible on at least the POSS II, and usually the POSS I, R-band plate material. None have either colours that are sufficiently red to be consistent with late-type M-dwarfs, or proper motions consistent with cluster membership.

\subsection{The colour-magnitude diagram}

Figure 1a plots the $\left(\mathrm{M}_{V},(\mathrm{~V}-\mathrm{I})\right)$ colour-magnitude diagram for M-dwarfs that are identified as Hyades members based on their proper motions (Reid, 1993, appendix The fainter stars with HST observations are identified separately in the figure. All of the binaries except RHY 240AB (the two lowest luminosity binary components, see paper I) have joint photometry, and most lie significantly above the main sequence. We have used the HST observations to estimate the relative contribution of each binary component to the ground-based V \& I data, and Figure 1b plots the results. We assume equal-mass components for each of the possible binaries listed in Table 1. Lacking high-resolution V-band data, we have plotted the primary stars at the joint photometry colours - in most cases, the components are of nearly equal-mass and the colours can be expected to be little-affected. We have estimated (V-I) colours for the secondary stars (excepting RHY $240 \mathrm{AB})$ based on the mean colour-magnitude relation.

The reduced scatter in Figure 1b is partly a result of these approximations. However, it is also clear that the main-sequence is noticeably narrower once due allowance is made for the composite nature of the HST binaries. There are a number of other obvious candidate binaries, notably RHy 390 at $\mathrm{M}_{V}=15.03$, (V-I)=3.83, but also amongst earlier-type Mdwarfs. We are currently obtaining high-resolution spectroscopic observations, using HIRES on Keck I, with the aim of identifying close stellar binaries amongst the lower-luminosity

\footnotetext{
${ }^{3}$ In addition to the late-type M-dwarfs cited in section 2.1, five stars listed as possible members by Reid (1993) have since been identified as field stars: vA 106 (G 7-150), G 7-116, RHy 61 (LP 474-1156), RHy 131 and RHy 158 (LP 415-1198).
} 
stars.

Until recently, the usual representation of the $\left(\mathrm{M}_{V},(\mathrm{~V}-\mathrm{I})\right)$ lower main-sequence was a linear relation. However, Leggett et al. (1994) first pointed out that the Hyades colour-magnitude relation exhibited significant non-linearities at fainter magnitudes. Gizis $\&$ Reid (1996) demonstrated that there was a substantial discontinuity in the absolute magnitude/TiO bandstrength relation at $\mathrm{M}_{V} \sim+12$; and Reid \& Gizis (1997) have shown that a break occurs at similar luminosities in the field-star $\left(\mathrm{M}_{V},(\mathrm{~V}-\mathrm{I})\right)$ lower main-sequence. Clemens et al. (1997) have argued that this feature is not due to atmospheric effects (i.e., structure in the effective temperature/colour relation), but rather reflects a change in the internal structure. Figure 1b plots the three-segment colour-magnitude relation derived by Reid \& Gizis (1997)] from V-and I-band photometry of Solar Neighbourhood stars. The higher-metallicity Hyades stars are redder than local field stars of the same absolute magnitude but, simply offsetting the nearby-star relation by 0.17 magnitudes in $(\mathrm{V}-\mathrm{I})$ to fit the Hyades main-sequence at $\mathrm{M}_{V}<11$ leads to a reasonable match to the general morphology of the data at fainter magnitudes. Clemens et al. (1997) have suggested that the change in slope at $\mathrm{M}_{V} \sim+12$ reflects a change in the mass-radius relation, perhaps as a result of the stars becoming fully convective. Whether this hypothesis is confirmed or not, cluster colour-magnitude diagrams offer the possibility of tracing the position of this break as a function of abundance and age. Reliable identification of such features, however, demands both accurate photometry and an adequate multiple-star census, as demonstrated by Figures $1 \mathrm{a}$ and $1 \mathrm{~b}$.

\section{The Binary Fraction}

Combining the present observations with the results from paper I, we have identified at least nine, and perhaps as many as twelve, binaries amongst our total sample of fifty-three Hyades M-dwarfs. This corresponds to a binary fraction, $\mathrm{f}_{\text {ovbs }}$ of between 17 and 23\%, with formal uncertainties of $\pm 7 \%$ through counting statistics. These estimates are consistent with the results derived in our preliminary analysis (Gizis \& Reid, 1995). Clearly, this represents a lower limit to the the overall binary fraction, $\mathrm{f}_{\text {tot }}$, since only companions with projected separations of at least 0.1 arcseconds can be resolved unambiguously by the PC. Indeed, as described in the previous section, the limiting magnitude for detecting a companion star varies with separation due to the underlying point-spread function of the primary star. Thus, transforming the observed binary fraction to an estimate of the total binary fraction requires a model for the semi-major axis distribution so that one can allow

for for unresolved systems at small angular separation. In paper I we combined an estimate 
of the detection efficiency as a function of radius with two representations of the semi-major axis distribution (the Duquennoy \& Mayon G-dwarf log-normal period distribution, and Fischer \& Marcy's (1992) results for field M-dwarfs) to derive the appropriate correction factor. Applying that factor to the value of $\mathrm{f}_{o b s}$ derived in paper I leads to an inferred $\mathrm{f}_{t o t}$ of $27 \pm 16 \%$, with the substantial uncertainties reflecting the small sample size. Our present data allow a modest improvement in the statistical accuracy.

We adopt a slightly different strategy in the analysis in the current paper. Theoretical models calculated by Burrows et al. (1993) predict that the hydrogen-burning limit in the Hyades corresponds to $\mathrm{M}_{b o l} \sim 12.8$. The predicted effective temperature is $\sim 2700 \mathrm{~K}$, comparable to that of GJ 1111, for which Leggett et al. (1996) derive an I-band bolometric correction of 0.3 magnitudes. This implies that the hydrogen-burning limit corresponds to $\mathrm{M}_{I} \sim 13.1$ magnitudes, 4 magnitudes fainter than the brightest stars in our sample. On the basis of our analysis of the HST images, we would expect to detect a companion with $\Delta I=4 \mathrm{mag}$ at a separation of $\rho \sim 0.28$ arcseconds, or 13.5 a.u. at a distance of 48 parsecs. Hence, our observations are capable of detecting all stellar companions with separations of between 13.5 and 825 a.u., where the upper limit is set by the field of view of the PC. These constraints define a complete sample of six binaries (RHy 49, 119, 221, 240, 246 and 377 Table 2), or a binary fraction of $11.3 \pm 4.6 \%$.

We can compare this statistic against the distribution of $\rho$ amongst the binaries in the 8-parsec sample defined by Reid \& Gizis (1997). In doing so, we take 825 a.u., corresponding to the semi-diameter of the PC field of view, as the upper limit to $\rho$ in the Hyades distribution. Gliese 644/VB 8 is the only local binary that lies outwith this limit. Limiting the calculation to the 78 systems where the brightest star has $\mathrm{M}_{v} \geq 8.0,11$ of the 24 known binary (or multiple) stars have components separated by at least 13.5 a.u. Thus, $\mathrm{f}_{o b s}$ is $14.1 \pm 4.2 \%$, consistent with our Hyades observations. It therefore follows that if the cluster binaries have a semi-major axis distribution that is comparable with the field M-dwarfs, then the implied $\mathrm{f}_{t o t}$ is $25 \pm 10 \%$. Macintosh et al. (1997) have pointed out that there is an apparent deficit of wide binaries $(\rho>100$ a.u.) amongst the Hyades stars when compared with the field-star sample. This might reflect disruption of such loosely-bound systems over the lifetime of the cluster.

Our results for the late-type Hyades stars can also be matched against the binary statistics for higher-mass cluster members. Patince et al (1997) have completed infrared speckle observations of 162 main-sequence Hyades stars with $K<8.5\left(\mathrm{M}_{K}<5, M_{V}<7\right)$, observations capable of detecting binary companions with $\rho>0.1$ arcseconds and $\Delta K<4$ magnitudes. Eleven of the 162 stars (or $6.8 \%$ of the sample) have resolved companions within the range of angular separation matching the HST PC observations. Since the 
K-band mass-luminosity relation can be approximated by $\mathrm{M}_{K} \propto 0.15 \times \log (\operatorname{mass})$, a magnitude difference of $\Delta K=4$ corresponds to a mass ratio of $\sim 0.25$. Thus, unlike our HST observations, in most cases the speckle data are incapable of detecting companions with masses close to the H-burning limit. One can allow for this incompleteness by adopting a mass function for the stellar companions. If that mass function matches the local field-star $\Psi(M)$, then the observed binary fraction underestimates the true binarity for this range of separations by a factor of two (see Patience et al., 1997 for full details). In that case, the $\mathrm{f}_{\text {obs }}$ amongst the higher-mass cluster-members for companions with $14<\rho<825$ a.u. is $13.5 \pm 4 \%$ - consistent with our observations of the late-type M-dwarfs in the cluster. With the relatively small number of stars in either sample, however, the statistical significance of this result is not strong,

\section{The mass-ratio distribution and brown dwarfs}

In their analysis of the later-type binaries amongst stars within 8-parsecs of the Sun, Reid \& Gizis (1997) argued that there was evidence for a bias towards equal-mass systems; that is, the hypothesis that both the primary and secondary star are drawn at random from the same mass function was not consistent with the observed distribution of mass-ratio, $\mathrm{q}=\frac{M_{2}}{M_{1}}$. We can test this result using our Hyades observations.

Table 2 lists mass estimates for each star in the resolved systems. These are based on the bolometric magnitudes, derived from $\mathrm{M}_{I}$ using the Leggett et al. (1996) bolometric corrections. For stars fainter than $\mathrm{M}_{b o l}=10$, we have used the 600-Myr X-models calculated by Burrows et al. (1993) to estimate masses, while masses for more luminous stars are estimated from an empirical relation calibrated by the Henry \& McCarthy (1993) nearby-star speckle data. For these calculations, we again limit the sample to the six binaries which fall in the régime where our data are capable of reaching the hydrogen-burning limit. The mass function for the local stars is well-represented by a power-law. $\Psi(M) \propto M^{-1}$ (Reid \& Gizis, 1997). Dynamical effects are likely to have modified the overall Hyades mass function, with preferential evaporation of lower-mass stars. However, while wide binaries may have been stripped, it is unlikely that the cluster dynamical evolution influences the companion-star mass distribution.

We have used Monte-Carlo techniques to model the expected mass-ratio distribution amongst binary stars, generating companions (mass $M_{C}$ ) to each star $\left(\operatorname{mass} \mathrm{M}_{*}\right.$ ) in the current sample, with $\Psi(M) \propto M^{-1}$ and $M_{*} \geq M_{C} \geq 0.08 M_{\odot}$. Figure 2 compares the predicted mass-ratio distribution, $\mathrm{F}(\mathrm{q})$, plotted as a cumulative distribution, against the observed distribution. With only six binaries in the latter sample, the statistical significance 
of the comparison is not overwhelming. However, three of the six stars are effectively equal-mass systems, and a Kolmogorov-Smirnoff test indicates that there is only a $10 \%$ chance of the observed distribution being drawn from the distribution predicted if the companion stars are selected from a $\Psi(M) \propto M^{-1}$ mass function. Thus, these data are consistent with the hypothesis that there is a bias toward equal-mass systems amongst M-dwarf binaries.

The HST imaging data are also capable of detecting higher-mass brown dwarfs. Three stars listed in Table 2 have companions with masses in the range 0.11 to $0.13 \mathrm{M}_{\odot}$, although all are clearly above the hydrogen-burning limit'. With a detection limit of $\Delta I$ $=5$ magnitudes for $\rho>0.31$ arcseconds, our deepest observations reach magnitude limits fainter than $\mathrm{I}=19.5$, corresponding to $\mathrm{M}_{I} \sim 16$, or $\mathrm{M}_{\text {bol }} \sim 15.5$. In the Burrows et al. (1993) models this luminosity corresponds to a brown dwarf of mass $0.05 \mathrm{M}_{\odot}$.

Since our observations extend beyond the hydrogen-burning limit, we can use these data to constrain the form of $\Psi_{S}(M)$, the secondary star mass function. If we adopt a functional form for $\Psi_{S}(M)$, we can calculate the expected relative number density of very low-mass (VLM) stars and substellar objects contributed by each star in our sample: that is, if $\Psi_{S}(M) \propto M^{-1}$ and the appropriate limiting magnitude corresponds to $\mathrm{M}_{I}=17$, or $M \sim 0.05 M_{\odot}$, then

$$
\frac{N\left(0.25 \geq M / M_{\odot}<0.1\right)}{N\left(0.1 \geq M / M_{\odot}>0.05\right)}=\frac{\int_{0.1}^{0.25} \Psi_{S}(M) d M}{\int_{0.05}^{0.1} \Psi_{S}(M) d M} \sim 1.3
$$

That is, with a mass function increasing as $\Psi(M) \propto M^{-1}$ towards lower masses, one predicts almost equal numbers of companions with masses between 0.05 and $0.1 \mathrm{M}_{\odot}$, and between 0.1 and $0.25 \mathrm{M}_{\odot}$. Given that each HST observation has a different limiting magnitude, we can determine the corresponding mass limit, and derive the appropriate ratio for that star. Summing the contribution from every star in the sample gives the expected relative numbers of stars and brown dwarfs detected in the present survey.

Since our observations are in the I-band, we need to determine the appropriate bolometric corrections to estimate the mass-limit corresponding to the limiting magnitude of each PC observation. A $0.055 \mathrm{M}_{\odot}$ Hyades brown dwarf is predicted to have a temperature of $\sim 1700 \mathrm{~K}$ (Burrows et al, 1993) and bolometric corrections remain poorly defined for objects of such low temperatures. However, GD 165B is estimated to have a temperature of

\footnotetext{
${ }^{4}$ The candidate substellar companion to RHy 281, reported in paper I, was re-observed by HST and is not a common proper-motion companion of the Hyades star.
} 
$\sim 1800 \pm 100 \mathrm{~K}$ (Tsuji et al., 1996), and Tinney et al. (1993)] have determined a bolometric correction of $\mathrm{BC}_{I}=1.75$ magnitudes for that star. Lacking other empirical results, we adopt a bolometric correction of $\mathrm{BC}_{I}=0.3$ magnitudes at $\mathrm{M}_{I}=13$ mag and assume a linear increase by 0.4 magnitudes per magnitude at fainter magnitudes.

We can used the mass-luminosity relation defined by the Burrows et al. (1993) models to transform the $\mathrm{M}_{b o l}$ detection-limit to a mass limit. However, these models are valid only if the Hyades has an age of 600 Myrs. While some recent analyses (e.g. Torres et al. (1996)] favour ages close to this value, other colour-magnitude diagram studies derive ages older by $\sim 300$ Myrs (Friel, 1995). Clearly, the older the cluster, the lower the luminosity achieved by brown dwarfs of a given mass. To take this uncertainty into account, we have also estimated mass-limits using the relation

$$
\frac{L}{L_{\odot}}=3.4 \times 10^{-6}\left(\frac{t}{10^{9}}\right)^{-1.3}\left(\frac{M}{20 M_{J}}\right)^{2.64}
$$

where $\mathrm{t}$ is the age in years, and $\mathrm{M}$ the mass in Jovian units. This relation, from Burrows \& Liebert (1993), is based on brown dwarf cooling models, and is expected to be valid for brown dwarfs older than $\sim 10^{8}$ years. There are relatively small differences between the Burrows et al. X-models and this mass-luminosity relation for an age of 600 Myrs.

Our calculations are therefore based on three brown dwarf mass-luminosity relations: the Burrows et al. X-models, and the Burrows \& Liebert (1993) relation for ages of 600 Myrs and 1 Gyr, taking the last as an upper limit to the cluster age. Table 3 lists the results, where $\alpha$ is the power-law exponent adopted for the mass function. We have divided the 0.25 to $0.05 \mathrm{M}_{\odot}$ mass range at 0.10 and $0.15 \mathrm{M}_{\odot}$, and compare the observed and predicted numbers above $(*)$ and below (VLM) the divide. The complete sample of six binaries defined above provide the empirical reference. While that reference sample is small in number, it is clear that, even for an age of $1 \mathrm{Gyr}$, a companion-star mass function $\Psi_{S}(M) \propto M^{-1}$ predicts almost $\sim 2 \sigma$ too many objects with masses below $0.15 \mathrm{M}_{\odot}$. If the Hyades age is close to 600 Myrs, then even a flat mass function $\left(\propto M^{0}\right)$ is inconsistent with the observations at the same level. These results, therefore, favour the hypothesis that both VLM dwarfs and brown dwarfs are infrequent companions to lower-mass M-dwarfs. 


\section{Conclusions}

We have completed our HST Planetary Camera search for lower-luminosity companions of a sample of fifty-three low-mass $\left(M<0.3 M_{\odot}\right)$ members of the Hyades cluster. Each observation is capable of detecting companions with masses at the hydrogen-burning limit where the projected angular separation is at least 0.28 arcseconds. Setting the outer radial limit at the circle circumscribed by the PC image, this corresponds to linear separations of from $\sim 14$ to 825 a.u. at the average distance of the Hyades cluster. In the case of the lowest luminosity stars, we expect to be able to detect brown dwarfs with masses as low as $0.05 \mathrm{M}_{\odot}$. However, within the annulus where our observations are complete, we identify only six stellar companions. The binary fraction is comparable with that observed amongst local M-dwarfs in the same range of separations and, as with the local stars, there is a statistically-significant preference for equal-mass systems and a scarcity of both very low-mass M-dwarf and brown dwarf companions.

This research was funded by HST grant GO-5353.01-93A and GO-06344.01-95A. This work is based partly on photographic plates obtained at the Palomar Observatory 48-inch Oschin Telescope for the Second Palomar Observatory Sky Survey which was funded by the Eastman Kodak Company, the National Geographic Society, the Samuel Oschin Foundation, the Alfred Sloan Foundation, the National Science Foundation grants AST84-08225, AST87-19465, AST90-23115 and AST93-18984, and the National Aeronautics and Space Administration grants NGL 05002140 and NAGW 1710. The first Palomar Observatory Sky Survey was funded by the National Geographic Society, and the Oschin Schmidt Telescope is operated by the California Institute of Technology and Palomar Observatory. 


\section{REFERENCES}

Burrows, A., Hubbard, W.B., Saumon, D. \& Lunine, J.I. 1993, ApJ, 406, 158

Burrows, A. \& Liebert, J. 1993, J. Rev. mod. Phys. 65, 301

Bryja, C., Humphreys, R.M. \& Jones, T.J. 1994, AJ, 107, 246

Clemens, J.C., Reid, I.N., Gizis, J.E. \& O'Brien, M.S. 1997, ApJ. submitted

Detweiler, H.L., Yoss, K.M., Radick, R.R. \& Becker, S.A., 1984, AJ, 1038

Duquennoy, A. \& Mayor, M. 1991, A\&A, 248, 485

Fischer, D.A. \& Marcy, G.W. 1992, ApJ, 396, 178

Friel, E.D. 1995, ARA\&A, 33, 381

Gliese, W. 1969, Veroff. Astr. Rechen-Instituts, Heidelberg, Nr. 22

Gliese, W. \& Jahreiss, H. 1979, A\&AS, 38, 423

Gizis J. \& Reid, N. 1995, AJ, 110, 1248

Gizis J.E. \& Reid, I.N. 1996, AJ, 111, 365

Griffin, R.F., Gunn, J.E., Zimmerman, B.A. \& Griffin, R.E.M. 1988, AJ, 96, 172

Gunn, J.E., Griffin, R.F., Griffin, R.E.M. \& Zimmerman, B.A. 1988, AJ, 96, 198

Henry, T.J. \& McCarthy, D.W. 1990, ApJ, 350, 334

Henry, T.J. \& McCarthy, D.W. 1993, AJ, 106, 773

Leggett, S.K., Allard, F., Berriman, G., Dahn, C.C. \& Hauschildt, P.H. 1996, ApJS, 104, 117

Leggett, S.K., Harris, H.C. \& Dahn, C.C. 1994, AJ, 108, 944

Luyten, W.J., Hill, G. \& Morris, S. 1981, Proper Motion Survey with the 48-inch Schmidt Telescope, LIX, Univ. Minnesota, Minneapolis

Macintosh, B.A., Zuckerman, B. \& Becklin, E.E. 1997, ApJ, in press

Marcy, G.W. \& Benitz, K. 1989, ApJ, 344, 441

Mason, B.D., McAlister, H.A., Hartkopf, W.I. \& Bagnuolo, Jr., W.G. 1993 AJ, 105, 220 
Patience, J., Ghez, A., Reid, I.N., Matthews, K.M. \& Weinberger, A. 1997, in preparation

Pels, G., Oort, J.H. \& Pels-Kluyer, H.A. 1975, A\&A, 43, 423

Reid, I.N. 1992, MNRAS, 257, 257

Reid, I.N. 1993, MNRAS, 265, 785

Reid, I.N., Hawley, S.L. \& Mateo, M. 1995, MNRAS, 272, 828

Reid, I.N., Gizis, J.E. 1997, AJ, 113, 2246

Schwann, H. 1991, A\&A, 243, 386

Simons, D.A., Henry, T.J. \& Kirkpatrick, J.D. 1996, AJ, 112, 2238

Stobie, R.S., Ishida, K. \& Peacock, J.A. 1989, MNRAS, 238, 709.

Tinney, C.G., Mould, J.R. \& Reid, I.N. 1993, AJ, 105, 1045

Torres, G., Stefanik, R.P. \& Latham, D.W. 1997, ApJ, 479, 268

Tsuji, T., Ohnaka, K., Aoki, W. \& Nakajima, T. 1996, A\&A, 308, L29

van Bueren, H.G. 1952, BAN, 11, 385 
Table 1. HST observations of candidate low-mass Hyads

\begin{tabular}{|c|c|c|c|c|c|c|c|c|}
\hline Name & $\mathrm{V}$ & $(\mathrm{V}-\mathrm{I})$ & $\mathrm{M}_{I}$ & comments & $\mathrm{M}_{I}(\mathrm{~A})$ & $\rho$ & $\theta$ & $\mathrm{M}_{I}(\mathrm{~B})$ \\
\hline \multicolumn{9}{|c|}{ Binaries } \\
\hline RHy 49 & 15.89 & 3.04 & 9.37 & $\mathrm{r}=49.7 \mathrm{pc}$ & 10.00 & $0^{\prime \prime} \cdot 36$ & 103 & 11.65 \\
\hline RHy 119 & 17.29 & 3.54 & 10.52 & 44.3 & 11.23 & 0.88 & 187 & 11.32 \\
\hline RHy 221 & 15.88 & 3.10 & 9.34 & 48.8 & 10.19 & 0.31 & 80 & 10.22 \\
\hline RHy 244 & 15.64 & 2.97 & 9.00 & 54.2 & 9.33 & 0.14 & 162 & 10.45 \\
\hline RHy 346 & 16.03 & 3.18 & 9.56 & 45.5 & 10.12 & 0.48 & 250 & 10.55 \\
\hline \multicolumn{9}{|c|}{ Binaries? } \\
\hline RHy 126 & 17.33 & 3.38 & 10.07 & $59.7 \mathrm{pc}$ & 11.25 & $\sim 0.06$ & 171 & 11.25 \\
\hline RHy 309 & 16.75 & 3.24 & 9.39 & 66.7 & 10.15 & $\sim 0.05$ & 57 & 10.15 \\
\hline RHy 391 & 16.42 & 3.35 & 9.63 & 48.8 & 10.35 & $\sim 0.08$ & 131 & 10.35 \\
\hline \multicolumn{9}{|c|}{ Single stars } \\
\hline RHy 23 & 16.18 & 3.01 & 9.62 & $51.3 \mathrm{pc}$ & & & & \\
\hline RHy 46 & 16.68 & 3.12 & 9.86 & 55.0 & & & & \\
\hline RHy 64 & 16.33 & 3.23 & 10.32 & 36.0 & & & & \\
\hline RHy 101 & 17.11 & 3.46 & 10.64 & 40.0 & & & & \\
\hline RHy 115 & 16.50 & 3.03 & 9.61 & 59.2 & & & & \\
\hline RHy 128 & 17.63 & 3.34 & 10.77 & 50.6 & & & & \\
\hline RHy 143 & 16.23 & 3.02 & 9.71 & 50.1 & & & & \\
\hline RHy 162 & 16.54 & 3.21 & 10.32 & 40.0 & & & & \\
\hline RHy 163 & 15.44 & 3.00 & 9.38 & 40.9 & & & & \\
\hline RHy 182 & 15.89 & 3.06 & 9.37 & 49.2 & & & & \\
\hline RHy 199 & 16.70 & 3.22 & 10.10 & 47.4 & & & & \\
\hline RHy 200 & 16.07 & 2.89 & 9.57 & 52.7 & & & & \\
\hline RHy 206 & 16.63 & 3.09 & 10.06 & 49.7 & & & & \\
\hline RHy 219 & 16.37 & 2.87 & 10.06 & 48.8 & & & & \\
\hline RHy 230 & 18.25 & 3.42 & 11.20 & 53.2 & & & & \\
\hline RHy 231 & 15.89 & 3.11 & 9.28 & 50.1 & & & & \\
\hline RHy 242 & 15.91 & 2.92 & 9.53 & 49.2 & & & & \\
\hline RHy 260 & 16.40 & 3.07 & 10.35 & 39.4 & & & & \\
\hline RHy 297 & 17.04 & 3.18 & 10.14 & 55.5 & & & & \\
\hline RHy 298 & 15.90 & 2.93 & 9.62 & 46.8 & & & & \\
\hline RHy 312 & 15.21 & 2.86 & 9.17 & 43.3 & & & & \\
\hline RHy 331 & 16.43 & 2.99 & 10.21 & 44.3 & & & & \\
\hline RHy 369 & 16.14 & 2.92 & 9.57 & 53.7 & & & & \\
\hline
\end{tabular}


Table 1 - Continued

\begin{tabular}{|c|c|c|c|c|c|c|c|c|}
\hline Name & V & $(\mathrm{V}-\mathrm{I})$ & $\mathrm{M}_{I}$ & comments & $\mathrm{M}_{I}(\mathrm{~A})$ & $\rho$ & $\theta$ & $\mathrm{M}_{I}(\mathrm{~B})$ \\
\hline RHy 376 & 15.80 & 2.90 & 9.52 & 47.4 & & & & \\
\hline RHy 399 & 15.09 & 2.92 & 9.16 & 40.0 & & & & \\
\hline RHy 402 & 17.77 & 3.25 & 10.59 & 61.1 & & & & \\
\hline B 804 & 19.27 & 3.87 & 11.96 & 48.8 & & & & \\
\hline & & & & Non-members & & & & \\
\hline RHy 77 & 17.37 & 2.81 & & $\mathrm{P}=19 \%^{1}$, binary & & & & \\
\hline RHy 80 & 17.94 & 3.17 & & $\mathrm{P}=3 \%$, no $\mathrm{H} \alpha$ & & & & \\
\hline RHy 110 & 16.78 & 3.03 & & $\mathrm{P}=8 \%$, triple & & & & \\
\hline RHy 271 & 17.86 & 3.09 & & $\mathrm{P}=3 \%$, no X-ray & & & & \\
\hline
\end{tabular}

${ }^{1}$ The formal probability, based on proper motion alone, that the star is a member of the Hyades cluster.

Note. - HST observations of binary stars were made on the following dates: RHy 49 - 25:01:96; RHy 119 - 15/02/96; RHy 126 - 19/11/95; RHy 221 - 23/02/96; RHy 244 - 13/09/95; RHy 309 - 03/02/96; RHy 346 - 25/10/96; RHy 391 04/02/96. 
Table 2. Binary star masses

\begin{tabular}{rrrcccc}
\hline \hline RHy & $\mathrm{M}_{I}(\mathrm{~A})$ & $\mathrm{M}_{I}(\mathrm{~B})$ & $\Delta($ a.u. $)$ & $M_{A}\left(\mathrm{M}_{\odot}\right)$ & $M_{B}\left(\mathrm{M}_{\odot}\right)$ & $\mathrm{q}$ \\
\hline 49 & 10.20 & 10.33 & 17.9 & 0.17 & 0.17 & 0.98 \\
119 & 11.04 & 11.32 & 39.0 & 0.13 & 0.11 & 0.88 \\
202 & 9.88 & 11.66 & 12.3 & 0.21 & 0.10 & 0.51 \\
221 & 10.08 & 10.11 & 15.1 & 0.19 & 0.18 & 0.99 \\
240 & 11.53 & 11.62 & 152.7 & 0.11 & 0.11 & 0.98 \\
244 & 9.18 & 10.45 & 7.6 & 0.25 & 0.16 & 0.65 \\
346 & 10.06 & 10.55 & 21.8 & 0.19 & 0.16 & 0.86 \\
371 & 9.26 & 10.59 & 6.8 & 0.25 & 0.21 & 0.70 \\
377 & 9.68 & 10.62 & 66.4 & 0.22 & 0.15 & 0.70 \\
\hline
\end{tabular}


Table 3. Predicted numbers of VLM stars and brown dwarfs

\begin{tabular}{lccccc}
\hline \hline & $\alpha$ & $\mathrm{N}\left(\mathrm{M}_{*}^{1}\right)$ & $\mathrm{N}\left(\mathrm{M}_{V L M}^{1}\right)$ & $\mathrm{N}\left(\mathrm{M}_{*}^{2}\right)$ & $\mathrm{N}\left(\mathrm{M}_{V L M}^{2}\right)$ \\
\hline & & & & & \\
Observations & & 6 & 0 & 4 & 2 \\
Analytic & 0 & 6 & 1.1 & 4 & 3.1 \\
600 Myr & -0.5 & 6 & 1.6 & 4 & 4.3 \\
& -1 & 6 & 2.2 & 4 & 5.9 \\
& -1.5 & 6 & 3.1 & 4 & 8.2 \\
Analytic & 0 & 6 & 0.4 & 4 & 2.3 \\
1 Gyr & -0.5 & 6 & 0.5 & 4 & 3.0 \\
& -1 & 6 & 0.7 & 4 & 3.9 \\
& -1.5 & 6 & 1.0 & 4 & 5.1 \\
Burrows et al & 0 & 6 & 1.8 & 4 & 4.4 \\
X-files & -0.5 & 6 & 1.9 & 4 & 4.6 \\
& -1 & 6 & 2.7 & 4 & 6.4 \\
& -1.5 & 6 & 3.7 & 4 & 9.0 \\
\hline
\end{tabular}

Note. - The relative numbers of companions within specific mass ranges predicted by power-law mass functions, compared with the observed number of companions (line 1).

$\alpha$ : the power-law index of the mass function

$\mathrm{N}\left(\mathrm{M}_{*}^{1}\right)$ : number of secondaries with $0.25 \geq \frac{M}{M_{\odot}}>0.10$

$\mathrm{N}\left(\mathrm{M}_{V L M}^{1}\right)$ : number of secondaries with $0.10 \geq \frac{M}{M_{\odot}}>0.05$

$\mathrm{N}\left(\mathrm{M}_{*}^{2}\right)$ : number of secondaries with $0.25 \geq \frac{M}{M_{\odot}}>0.15$

$\mathrm{N}\left(\mathrm{M}_{V L M}^{2}\right)$ : number of secondaries with $0.15 \geq \frac{M}{M_{\odot}}>0.05$ 

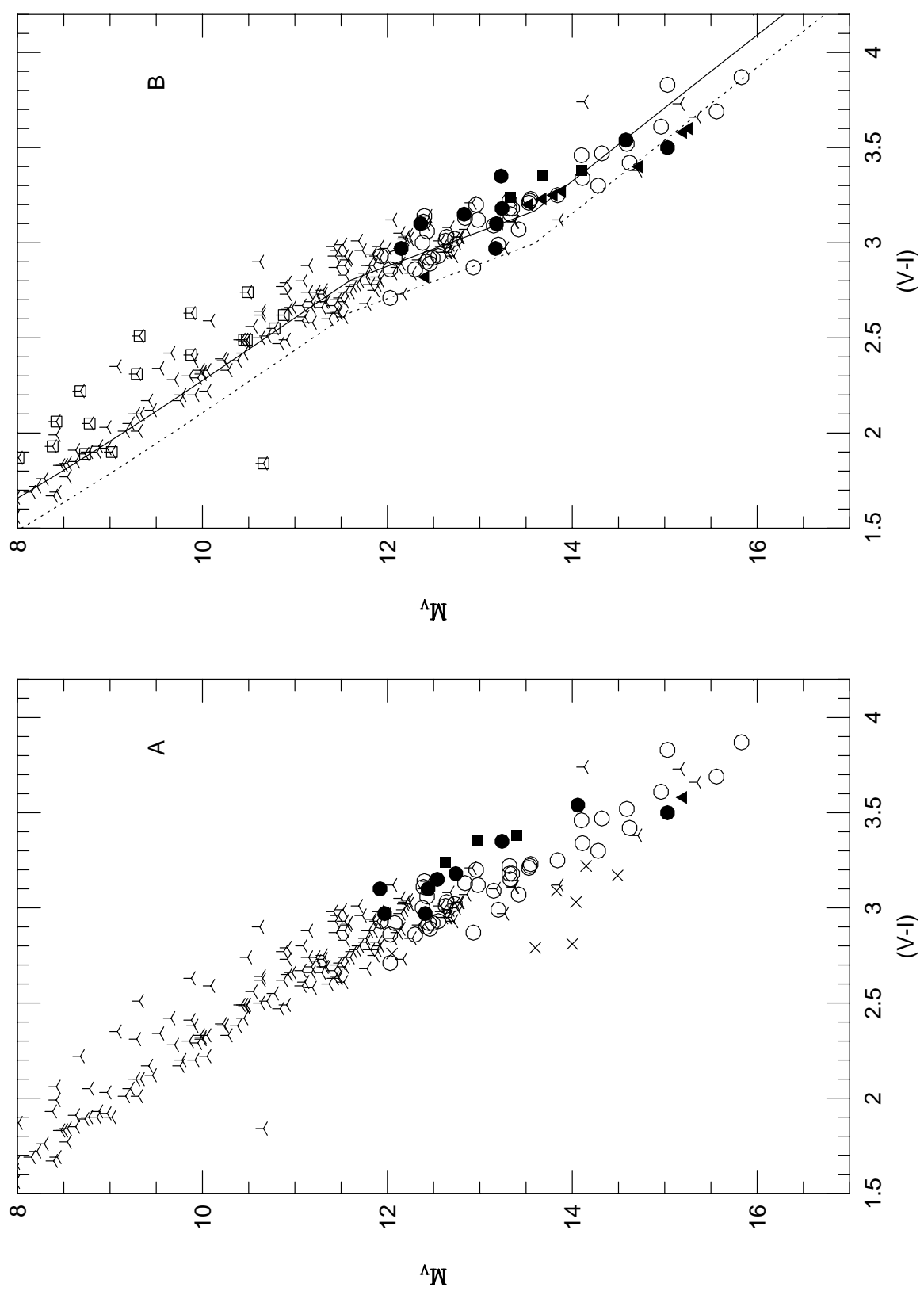

Fig. 1. - The Hyades colour-magnitude diagram. a) Open circles are Hyades members with no resolved companion in the HST images; solid circles are stars with resolved companions; solid squares are possible binaries; four-point crosses are stars identified as nonmembers; and three-point crosses mark data for cluster members with no HST observations. The solid triangle marks RHy 240B, the only secondary star with separate ground-based photometry. The VI photometry is taken directly from Reid (1993) and Leggett et al. (1994). b) The symbols have the same meaning as in figure 1a, save that the open squares identify known spectroscopic binaries amongst the brighter stars, and we have used the HST observations to estimate deconvolved magnitudes and colours for the binaries. The primary stars are plotted as solid circles while the secondaries are plotted as solid triangles. The dotted line plots the three-segment relation matched to the local stars by Reid \& Gizis (1997). The solid line plots the same relation offset by 0.17 magnitudes. 


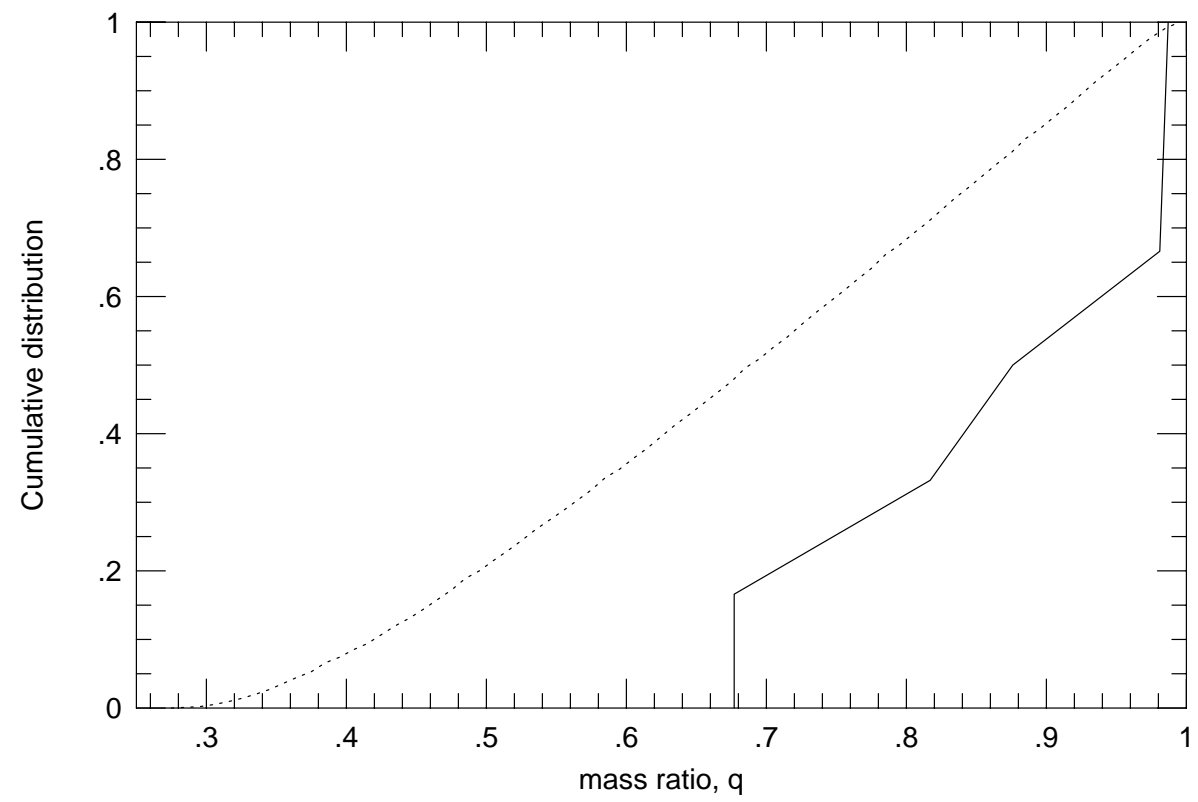

Fig. 2.- Mass-ratio distribution: the dotted line is the expected mass-ratio distribution for the HST sample if companion stars have a mass function which varies as $\mathrm{M}^{-1}$. The calculations were truncated at the hydrogen-burning limit. The solid line is the observed mass-ratio distribution of the six binaries in our complete sample. A Kolmogorov-Smirnoff test shows that there is only a $10 \%$ chance of the latter distribution being drawn from the predicted distribution. 